\title{
点云场景下基于结构感知的车辆检测
}

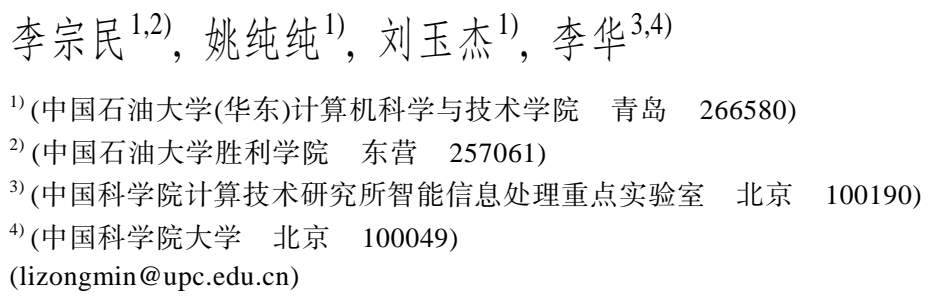

摘 要: 在自动驾驶领域, 计算机对周围环境的感知和理解是必不可少的. 其中, 相比于二维目标检测, 三维点云目 标检测可以提供二维目标检测所不具有的物体的三维方位信息, 这对于安全自动驾驶是至关重要的. 针对三维目标 检测中原始输入点云到检测结果之间跨度大的问题, 首先, 提出了基于结构感知的候选区域生成模块, 其中定义了 每个点的结构特征, 充分利用了三维点云目标检测数据集提供的监督信息, 通过预测该特征, 网络可以学习到更具 有鉴别能力的特征，从而提高候选框的生成质量；其次，将该特征加人到候选框微调阶段中，使得点云上下文特征 和局部特征更加丰富. 在三维点云目标检测数据集进行了实验, 结果表明, 文中方法能够在增加极少计算量的前提 下，在候选区域生成阶段使用 50 个候选框 0.7 的 IoU 阈值下，提高超过 $13 \%$ 的召回率; 在候选框微调阶段, 3 种难度 目标框的检测效果均有明显提升, 表明了该方法对三维点云目标检测的有效性.

关键词: 三维点云目标检测; 结构特征; 候选区域生成网络

中图法分类号: TP391.41 DOI: 10.3724/SP.J.1089.2021.18368

\section{Vehicle Detection Based on Structure Perception in Point Cloud}

\author{
Li Zongmin ${ }^{1,2)}$, Yao Chunchun ${ }^{1)}$, Liu Yujie ${ }^{1)}$, and Li Hua ${ }^{3,4)}$ \\ 1) (College of Computer Science and Technology, China University of Petroleum, Qingdao 266580) \\ ${ }^{2)}$ (Shengli College of China University of Petroleum, Dongying 257061) \\ 3) (Key Laboratory of Intelligent Information Processing, Institute of Computing Technology, Chinese Academy of Sciences, Beijing 100190) \\ 4) (University of Chinese Academy of Sciences, Beijing 100049)
}

\begin{abstract}
In the field of automatic driving, computer perception and understanding of the surrounding environment is essential. Compared with 2D object detection, 3D point cloud object detection can provide the three-dimensional information of the object that the 2D object detection does not have. In order to solve the problem of large disparity between the original input point cloud and the detection result in 3D object detection, a region proposal generation module based on structure awareness is proposed, in which the structural features of each point are defined, and the supervision information provided by the 3D point cloud object detection dataset is fully utilized. The network can learn more discriminative features to improve the quality of proposals. Secondly, the feature is added to the proposal fine-tuning stage to enrich the context features and local features of point cloud. Evaluated on KITTI 3D object detection dataset, in the region proposal
\end{abstract}

收稿日期: 2020-05-07; 修回日期: 2020-08-01. 基金项目: 国家自然科学基金(61379106, 61379082, 61227802); 山东省自然科学 基金(ZR2013FM036，ZR2015FM011); 中央高校基本科研业务费专项资金(18CX06050A). 李宗民(1965一), 男, 博士，教授，博士生 导师, CCF 会员, 主要研究方向为计算机图形学、图像处理、模式识别、科学计算化; 姚纯纯(1996一), 男, 硕士研究生, 主要研究方 向为三维点云目标检测; 刘玉杰(1971一), 男, 博士, 副教授, CCF 会员, 主要研究方向为计算机图形图像处理、多媒体数据分析、多 媒体数据库; 李华(1957一), 男, 博士, 研究员, 博士生导师, CCF 会员, 主要研究方向为计算机图形图像处理. 
generation stage, under the IoU threshold of 0.7 , using 50 proposals, there is a more than $13 \%$ increase in the recall rate compared to previous results. In the proposal fine-tuning stage, the detection results of the 3 difficulty levels objects is obviously improved, indicating the effectiveness of the proposed method for 3D point cloud object detection.

Key words: 3D point cloud object detection; structure feature; region proposal network

近年来，深度学习在二维计算机视觉中取得 了很多突破成就，包括图像分类 ${ }^{[1-2]}$ 、图像检索 ${ }^{[3]}$ 、 目标检测 ${ }^{[4]}$ 与实例分割 ${ }^{[5]}$ 等。但在很多实际应用, 如自动驾驶、机器人导航和增强现实等场景中, 仅 仅对二维世界进行感知是远远不够的. 其中三维 目标检测对于实现安全自动驾驶尤为重要，因为 它可以提供车辆和行人等在三维世界中的位置、大 小和朝向等信息. 激光雷达性能的提高和价格的 降低、GPU 设备的普及以及未来 $5 \mathrm{G}$ 数据传输能力 的提高, 很大程度上降低了人们获取和处理三维 点云数据的门槛. 然而, 相对于研究广泛的二维目 标检测，三维点云目标检测的文献相对较少，当前 迫切需要基于点云的目标检测方法. 在本文中, 实 验验证了基于结构感知的候选区域生成网络在三 维点云目标检测任务中的作用.

通过激光雷达获取的原始三维点云，仅包含 点 $x y z$ 坐标和反射强度，区别于图像信息. 直接处 理点云会面临点云数据量大、无序和不规则等问 题，因此目前很多的三维点云目标检测方法都将 点云投影到鸟瞰图 ${ }^{[6-9]}$ 、前景视图 ${ }^{[10]}$ 等来表示，再 将投影后的特征图输人到深度网络中, 以减少数 据处理的难度, 并且利用得到广泛研究的二维卷 积神经网络, 使得点云三维目标检测成为可能, 并 在中等难度下取得了超过 0.6 的平均精度(average precision, AP), 在一定程度上促进了点云三维目标 检测领域进一步研究的开展. 但点云数据在投影 过程中会不可避免地会导致分辨率下降、丢失三维 结构信息，而三维结构信息对于三维目标的精确 检测是至关重要的. 还有一些方法将点云场景栅 格化为体素 ${ }^{[11-13]}$, 用 0,1 或进一步提取该体素的特 征来表示，这样的方法虽然可以方便地使用三维卷 积网络进行特征提取, 并且保留了大部分空间相对 位置关系，但仍会造成信息丢失、计算量大等问题.

为了减少点云量化过程中出现的信息丢失等 问题, Charles 等 ${ }^{[14]}$ 提出了 PointNet, 以对称函数的 方法聚合点云全局特征; 又提出 PointNet $+{ }^{[15]}$ 对 采样点的邻域进行编码, 从而学习到点云局部结构
特征, 为实现直接处理点云数据进行三维点云目标 检测提供了思路. 后续工作 F-PointNet ${ }^{[16]}$ 首先利用 现有二维目标检测框架在图像中检测到物体，然 后根据检测结果在三维空间中限定 1 个视雉区域, 在视雉区域中使用 PointNet/++模型 ${ }^{[14-15]}$ 处理点云, 获得物体的三维空间信息，包括位置、尺寸和朝向 信息. 但这种方法对于传感器(相机、激光雷达)之 间同步精度要求比较高, 并且无法利用三维信息 来生成候选框. 还有文献直接处理点云, 不需要输 人其他额外信息. 如 PointRCNN ${ }^{[17]}$ 将三维点云目 标检测分为 2 个阶段，第 1 阶段在生成候选框的同 时预测每个点的语义特征; 第 2 阶段结合第 1 阶段 预测的特征, 对候选框进行微调得到最终检测结 果, 其精度比较高. 但其没有很好地解决原始点云 和目标检测结果之间差别较大的问题，导致生成 的候选框不够精确. 本文在此基础上提出了结构 感知模块, 它充分利用了标注框带来的监督信息 和点云的稀疏性, 在点云原始特征和三维点云目 标检测结果之间搭建了桥梁，使得候选区域生成 网络的学习更加容易, 最终提高了候选框的召回 率和最终三维点云目标检测精度。

\section{1 相关工作}

\section{1 基于投影的方法}

由于在自动驾驶场景中处理点云需要解决点 云大规模、无序性和不规则等问题，而二维的目标 检测已经得到了广泛且深人的研究, 因此很多方 法将三维点云投影到平面，再使用当前成熟的图像 目标检测框架得到二维的检测结果, 或再投影回三 维得到三维目标. 其中代表性的工作有 BirdNet $^{[8]}$ 和 PIXOR ${ }^{[9]}$, 它们将点云投影成鸟瞰图; 而 $\mathrm{FVNet}^{[10]}$ 将点云投影到前景视图. 忽略点云场景固有的高 度或深度本身会丢失大量三维信息, 而三维信息 对于目标的精确定位是不可或缺的. 同时, 若投影 视图分辨率太高，则会导致后续处理计算量大; 反 之，将会丢失更多细节信息. 


\section{2 基于体素的方法}

由于点云数据是三维数据, 因此将点云量化 为体素, 再使用三维的卷积神经网络进行特征提 取和生成候选框也是一种常见方法. 然而点云仅 占有整个空间的很小一部分，在将整个场景量化 为体素的过程中, 这些空白的区域也会被量化. VoxelNet ${ }^{[11]}$ 将点云进行体素化，每一个体素内部 使用 PointNet ${ }^{[14]}$ 提取到 1 个特征向量，整个空间被 表示为 4 维的张量, 再输人到卷积中间层, 最终候 选区域生成网络(region proposal network, RPN)产 生三维点云目标的检测结果. 传统的三维卷积会 带来计算量大的问题, 很大一部分原因在于需要对 空白位置进行卷积. 为了减少冗余计算, SECOND ${ }^{[12]}$ 中使用子流形稀疏卷积 ${ }^{[18]}$, 在点云量化为体素的 过程中, 用字典数据结构存储非空体素的信息, 仅 在非空体素执行卷积操作; 且后续子流形稀疏卷 积过程中不会降低稀疏度, 这极大地减少了三维 卷积操作的计算量和三维场景体素化后的存储空间 需求. 尽管如此, 将点云量化为体素仍会不可避免 地带来信息丢失, 很难得到更为精细的检测结果.

\section{3 图像与点云融合的方法}

在自动驾驶任务中, 点云数据提供了三维结 构信息，而图像提供了丰富的纹理特征，所以很 多方法尝试融合多种模态的信息进行三维目标检 测. 其中, MV3D ${ }^{[19]}$ 将点云投影到鸟瞰图，并且训 练了一个区域生成网络, 用来生成候选区域。对 于每个候选区域，融合来自鸟瞰图、图像和点云 的前景视图来生成三维的目标框. $\mathrm{AVOD}^{[20]}$ 对 $\mathrm{MV} \mathrm{D}^{[19]}$ 的特征融合进行了改进, 它在候选区域 生成网络阶段进行多模态特征融合，生成了更高 质量的候选框.

F-PointNet ${ }^{[16]}$ 利用成熟的二维目标检测网络 直接从图像中得到二维目标框, 然后利用这些目 标框和相机参数在点云中获得 1 个视雉, 将目标限 定在视雉中，极大地缩小了搜索范围; 然后利用 PointNet/ $++^{[14-15]}$ 抽取视雉中点云的特征, 得到三 维目标的方位、尺寸和朝向信息. 然而这种方法依 赖于二维目标检测框架，并且无法充分地利用三 维信息来生成候选框，在一些极端天气和遮挡比 较严重等情况下可能会检测不到物体，而这些物 体无法在后续流程中被检测出来.

\section{4 直接处理点云的方法}

StarNet ${ }^{[21]}$ 直接在点云中进行最远点采样 (farthest point sampling, FPS), 确保采样点在空间 上分布均匀; 然后在每个采样点周围形成若干个
候选框, 以图卷积网络提取点云特征, 并产生最终 的检测结果. 但这样做缺乏对整个场景的感知, 会 在很多非目标区域生成候选框。Qi 等 ${ }^{[22]}$ 借鉴 Hough 变换的思想, 利用场景中的每个点向物体 中心进行投票，再学习投票后点的特征和局部几 何信息，从而生成高质量的候选框. 由于自动驾驶 场景中背景点占绝大多数, Hough 投票的方法仅用 在室内场景的三维点云目标检测.

PointRCNN ${ }^{[17]}$ 将三维点云目标检测分为 2 个阶 段. 首先是候选区域生成网络, 它利用 PointNet $++^{[15]}$ 对整个场景分割, 同时进行前景分割和候选框的 生成. 在第 2 阶段的微调网络中, 结合第 1 阶段预 测的语义特征和点云局部特征等对候选框进行微 调, 得到高质量的检测框. 点云中缺失纹理信息, 要获得物体精确的三维方位信息, 如何仅利用点 云数据获得更细粒度的局部特征尤为重要. 虽然 PointNet $+{ }^{[15]}$ 中存在对邻域编码的模块, 但没有 与之直接对应的监督信息, 网络需要直接从点云 中获得尺寸、位置和旋转角度信息, 存在语义鸿沟. 本文在 PointRCNN 模型 ${ }^{[17]}$ 的基础上加人了结构感 知模块, 在点云原始特征和三维目标信息之间建 立了桥梁，增强了每个点的表征能力，减少了网络 端到端学习的难度. 而且本文方法几乎没有增加 计算量和存储空间, 在候选框生成质量上得到了 极大提升.

\section{2 本文算法}

\section{1 问题定义}

在三维点云目标检测系统中，给定输人集 $P=\left\{p_{i}\right\}_{i=1}^{n} \subset \mathbb{R}^{3}$ 包含 $n$ 个点, 每个点用 3 个维度的 特征 $x y z$ 表示. 输出集可以表示为 $V=\left\{v_{i}\right\}_{i=1}^{k} \subset \mathbb{R}^{7}$, 共有 $k$ 个物体，每个物体可以表示为 $v=$ $\left(c_{x}, c_{y}, c_{z}, h, w, l, \theta\right)$. 其中, $c_{x}, c_{y}, c_{z}$ 表示物体的中 心坐标; $h, w, l$ 分别表示物体的高度、宽度和长度; $\theta$ 表示物体在鸟瞰图中的朝向.

\section{2 数据处理}

如图 1 所示, 前景视图为 KITTI数据集 ${ }^{[23]}$ 采集 车辆上方彩色相机前方所对应的视图, 仅在摄像 头能拍摄到的范围内有标注信息, 而原始点云是 围绕车辆 $360^{\circ}$ 采集的. 为了减少假负样本的数量, 需要将前方视野以外的点云进行剔除, 即删除图 1 中白色标志的点云, 保留黄色标志的点云. 每个场 景保留下来的点云数量是不一致的, 为了统一输人 
到 PointNet $++{ }^{[15]}$ 中, 对整个点云重采样到 16384 个 点. 重采样的方法如下: 在 KITTI 数据集 ${ }^{[23]}$ 中, 距 离采集设备远的物体被采集的点云较少, 若场景 中的点云数量大于 16384 , 则仅对距离采集设备 $40 \mathrm{~m}$ 以内的点云进行随机下采样, 以确保不会对 远距离的点云进行下采样; 若整个场景点云数量不 足 16384 , 则将对整个场景中点云进行随机上采样.

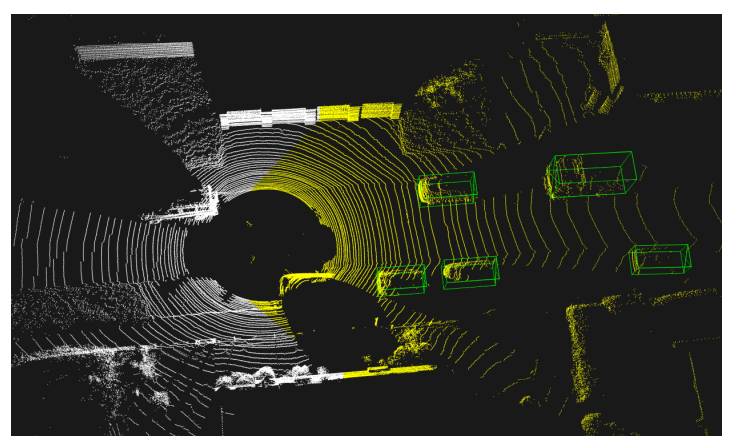

图 1 点云数据剔除示例

\section{3 网络模型}

本文网络模型以点云为输人, 输出为场景中 车辆的三维目标框和朝向信息. 将三维点云目标 检测分为如图 2 所示 2 个阶段，第 1 阶段的目标为

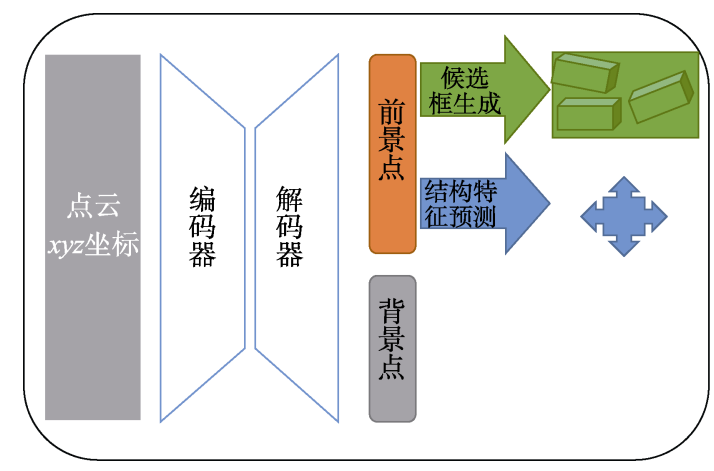

生成高质量的候选框, 采用 PointNet $++^{[15]}$ 编码处 理整个场景的 16384 个点云. 相对于 PointNet ${ }^{[14]}$, PointNet++可以对点的局部信息进行聚合. 在 PointNet++中有 4 个特征聚合模块, 将关键点的数 量从 16384 不断下采样到 4096, 1024, 256, 64. 每 一个特征聚合模块又由 3 层网络组成, 分别是采样 层、分组层和 PointNet 层. 其中, 采样层的作用是 在上一层的输人点云中使用 FPS 采集固定数量的 点. 随机采样的缺点是在点云密集的地方采样也 密集，导致采样点在空间分布上不均匀, FPS 方法 能够采集到在空间分布更均匀的点. 分组层以采 样点为中心, 以一定半径划分邻域, 其中的点云随 机重采样一定数量作为采样点的邻近点. PointNet 层使用全连接处理采样点的局部邻近点, 得到采 样点的局部特征向量. 下一层中, 以上一层的采样 点的空间位置和其对应特征向量为输人, 进一步 聚合特征. 层级越高, 采样点感受野越大, 特征越 抽象. PointNet++解码器由 4 个特征传播层组成, 采样点的数量从 64 不断上采样到 256, 1024,4096 , 16384 ; 在上采样的过程中, 插人点的位置是由下 采样过程确定的. 最终输出的 16384 个点与输人 的点云坐标相同, 但特征更丰富.

图 2 本文三维点云目标检测网络结构图

不同于二维目标检测，三维点云目标检测中 物体会保持真实尺寸, 三维目标在真实场景中的 位置不会因为采集设备的位置而改变，并且由于 点云是稀疏的，物体之间交叉的情况很少，标注框 内的点云基本都属于该物体. 因此三维标注框提 供了 2 个额外的监督信息: (1) 语义特征, 标注框 中的点云都是前景点, 其他点为背景点; (2) 结构 特征, 标注框内的点云在物体相对于物体中心的 各方向比例具有旋转不变性、平移不变性, 即无论 该物体旋转、平移, 某点的相对位置不变. 本文称 之为每个点的结构特征, 定义为

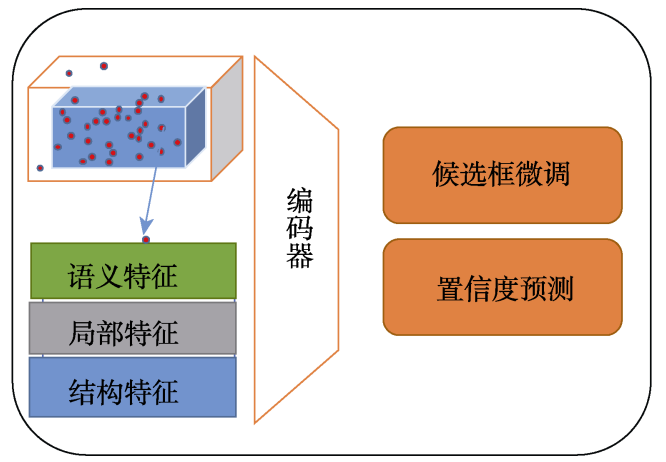


得到每个点的结构特征 $F_{\text {structure }}$.

\section{4 结构信息预测}

通常，距离采集设备的物体获得的点非常少， 并且遮挡问题严重，导致物体被采集的点云数量 少、不完整. 从极少量的点云中恢复物体的三维信 息是十分困难的，为了解决这个问题，Shi 等 ${ }^{[17]}$ 发 现数据集中标注框提供了区分前景点云和背景点 云的信息, 即位于标注框内的点云为前景点, 其他 的点都是背景点. Shi 等 ${ }^{[17]}$ 利用这个特点, 对整个 点云进行语义分割, 仅在前景点生成候选框，提高 了候选框的生成质量. 但仅有点云的前景信息是 不够的, 如图 3a 所示, 差异很大的若干候选框可 能包含同样的前景点, 如果仅能分辨哪些点是前 景点, 很难去除穴余的候选框. 本文提出的结构特 征可以很好地提供更细粒度的特征，在判断每个 点属于车的哪一部分的情况下 (如图 3b 中车前部 点云), 能够去除大量多余的候选框, 从而进一步 提高候选框生成的质量和检测精度.

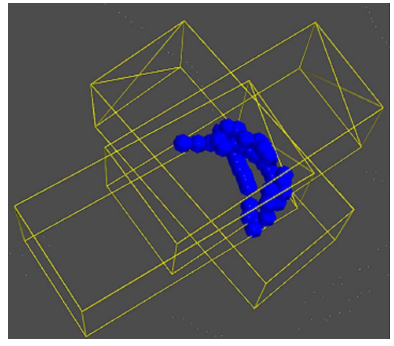

a. 包含点云的若干候选框

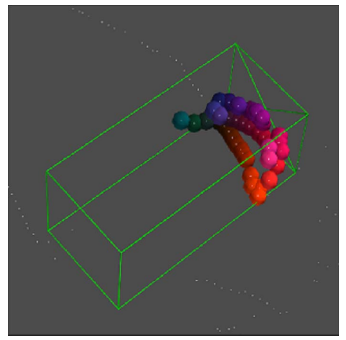

b. 真实目标框
图 3 利用结构特征过滤多余候选框示意图

每个场景中 16384 个点经过编码器解码器后, 会同时输出 3 个结果: 语义特征, 即每个点是前景 点或是背景点; 结构特征 $F_{\text {structure }}$; 表示 1 个候选框 的 7 个值为 $\left(c_{x}, c_{y}, c_{z}, h, w, l, \theta\right)$. 在 PiontRCNN ${ }^{[17]}$ 中, 候选区域生成阶段仅输出每个点的语义特征和候选 框. 统计得到前景点的数量和背景点的数量比值 小于 $1 / 10$, 如果直接使用交叉熵损失函数, 网络会 倾向于将所有点判断为背景点. 故本文使用 Focal Loss 损失函数 ${ }^{[4]}$ 来缓解前景点背景点比例悬殊的 问题. Focal Loss 损失函数定义为

$$
\begin{aligned}
L_{\mathrm{focal}}\left(p_{t}\right) & =-\alpha_{t}\left(1-p_{t}\right)^{\gamma} \log \left(p_{t}\right), \\
p_{t} & = \begin{cases}p, & \text { 对于前景点 } \\
1-p, & \text { 其他 }\end{cases}
\end{aligned}
$$

其中, 采用 $\mathrm{Lin}$ 等 $^{[4]}$ 使用的超参数, 设置 $\alpha_{t}=0.25$, $\gamma=2$. 虽然每个点都会输出表示结构特征和候选
框的值, 但仅对前景点对应的结构特征和候选框 产生损失. 本文使用二元交叉熵损失函数计算结 构特征损失.

第 1 阶段生成的高质量候选框，在第 2 阶段中 进一步微调. 从每一个候选框中随机采样 512 个 点, 使用候选区域生成阶段预测的语义特征、结构 特征和局部特征送人 PointNet ${ }^{[14]}$. 第 2 阶段网络模 型包括 3 个信息聚合层, 大小分别为 $128,32,1$; 最 终得到一维特征向量, 其输出包括车辆的置信度 和候选框的微调值.

\section{3 实验结果及分析}

\section{1 数据集介绍}

本文方法使用 KITTI 数据集 ${ }^{[23]}$ 进行三维点云 目标检测实验. 该数据集根据物体到采集设备的 距离、遮挡程度分为 3 个难度级别: 简单、中等和 困难. 物体有车辆、行人和自行车 3 个主要的类别. 由于车辆类别有最多的训练样本, 本文仅在车辆 类别上进行实验, 在其他类别上进行实验不需要 对现有流程进行修改. 公开的训练数据集被分为 训练集和验证集, 场景数量分别为 3712 和 3769 . 使用召回率评估生成候选框的质量, 使用 AP 评估 三维目标的精度.

\section{2 实验设置}

本文对候选区域生成网络和候选框微调网络 单独进行训练. 使用在线数据增强方式, 数据增强 方法包括对整个场景的旋转、平移、缩放和镜像翻 转, 在 PointRCNN ${ }^{[17]}$ 中还使用了目标框增强, 即将 若干候选框放置到其他场景中, 但这样可能带来一 些不合理的情况以及增加训练时间. 本文没有采用 此数据增强方式, 实验对比为 PointRCNN ${ }^{[17]}$ 不使用 此项数据增强的结果. 在训练过程中, 候选区域生 成网络使用 Adam 优化器, 学习率设置为 0.0002 共训练 200 周期. 由于加人结构特征后能够生成更 高质量的候选框, 因此与 PointRCNN ${ }^{[17]}$ 相比, 本 文使用了更高的非极大值抑制(non maximum suppression, NMS)阈值 0.88 来过滤掉低质量候选框 (PointRCNN 中使用 0.85 作为 NMS 阈值). 选择其 中交并比(intersection over union, IoU) 为 0.5 时召回 率最高的模型生成候选框, 用来训练第 2 阶段. 在 每个场景中保留 512 个候选框, 为了使训练更高 效, 在第 2 阶段中, 随机选择 64 个候选框用以训练 网络. 如果候选框和真实框的 IoU 超过 0.6, 则被 认为是正样本; IoU 低于 0.45 , 则被认为是负样本, 
其他 IoU 的样本在训练时被忽略. 第 2 阶段以学习 率 0.0002 训练 50 周期, 采用候选框的特征、结构 特征和局部特征等输人网络. 本文使用的硬件设 备为 $4 \times P 100$ 显卡, 每个显卡有 $16 \mathrm{~GB}$ 显存.

\section{3 实验结果与分析}

第 1 阶段为候选区域生成阶段, 其目的是生成 高召回率的候选框，并且输出具有区分力的特征 提供给第 2 阶段微调, 以产生更为精细的检测框结 果. 针对 PointRCNN ${ }^{[17]}$, 本文主要优化的是候选 区域生成阶段，对候选区域生成网络的召回率进 行了实验对比, 结果如表 1 所示. 表 1 中为在车辆 类别中等难度数据集上、不同数量的候选框 IoU 阈 值分别是 0.5 和 0.7 情况下的召回率. (注：由于不 明确文献[17]中召回率采用的 NMS 阈值, 为了公 平比较, 本文使用在 $\mathrm{IoU}=0.5$ 条件下使召回率最大 时的 NMS 阈值，使用文献[17]训练好的模型，列 出的 PointRCNN ${ }^{[17]}$ 召回率结果绝大部分要优于原 文献[17]中的结果). 本文方法几乎在所有的候选 框数量和 IoU 阈值下超过了对比的基准方法. 本文 方法仅仅需要 50 个候选框, 就可以在 $\mathrm{IoU}=0.5$ 的 阈值下得到 $96.93 \%$ 的召回率，在 $\mathrm{IoU}=0.7$ 的阈值 下得到 $73.39 \%$ 的召回率，与本文的基准线 PointRCNN 相比，召回率提高了 $13.16 \%$ ，且使用 40 个候选框得到了 PointRCNN ${ }^{[17]}$ 使用 100 个候选 框才可以达到的召回率. 并且在不同 IoU 阈值下, 本文方法的召回率提高了 $3.93 \%$ 24.14\%, 证明了 提出的预测结构特征方法能够使候选框生成网络 生成更高质量的候选框.

表 1 不同模型候选框和 IoU 下召回率对比 \%

\begin{tabular}{|c|c|c|c|c|c|c|}
\hline \multirow{2}{*}{$\begin{array}{c}\text { 候选框 } \\
\text { 数量 }\end{array}$} & \multicolumn{4}{|c|}{$\mathrm{IoU}=0.5$} & \multicolumn{2}{|c|}{$\mathrm{IoU}=0.7$} \\
\hline & $\mathrm{MV3D}^{[19]}$ & $\operatorname{AVOD}^{[20]}$ & $\begin{array}{c}\text { Point- } \\
\text { RCNN }^{[17]}\end{array}$ & 本文 & $\begin{array}{c}\text { Point- } \\
\text { RCNN }^{[17]}\end{array}$ & 本文 \\
\hline 10 & & 86.00 & 86.97 & 90.06 & 33.29 & 57.43 \\
\hline 20 & & & 92.58 & 94.60 & 40.29 & 61.92 \\
\hline 30 & & & 94.84 & 95.96 & 50.07 & 66.70 \\
\hline 40 & & & 95.71 & 96.47 & 52.44 & 70.66 \\
\hline 50 & & 91.00 & 96.30 & 96.93 & 60.23 & 73.39 \\
\hline 100 & & & 97.54 & 97.75 & 70.38 & 79.90 \\
\hline 200 & & & 98.05 & 98.11 & 74.99 & 84.96 \\
\hline 300 & 91.00 & & 98.24 & 98.20 & 82.80 & 86.73 \\
\hline
\end{tabular}

注. 加粗表示列出方法在相同 IoU 和候选框数量下的最 高精度.

本文为了验证提出的结构特征可以为候选框 微调提供更有区分力的特征, 将最终的检测结果 与当前的先进算法在 KITTI 的验证集 ${ }^{[23]}$ 上进行对
比，如表 2 所示. 精度数据来源于文献[17](本文没 有使用目标框增强，对比的 PointRCNN ${ }^{[17]}$ 也是没 有使用目标框增强的结果). 从结果中可以看出, 本文方法比 PointRCNN ${ }^{[17]}$ 精度有了明显提升，在 3 个难度等级(简单、中等和困难)上精度分别提升 $0.63 \%, 0.37 \%, 0.75 \%$. 说明了本文方法不仅能够提 高候选框的生成质量, 而且可以提高最终的检测 结果准确性.

表 2 三维点云目标检测结果 AP 对比 $(\mathbf{I o U}=\mathbf{0 . 7}) \%$

\begin{tabular}{|c|c|c|c|}
\hline \multirow{2}{*}{ 方法 } & \multicolumn{3}{|c|}{ 三维目标检测难度 } \\
\hline & 简单 & 中等 & 困难 \\
\hline MV3D $^{[19]}$ & 71.29 & 62.68 & 56.56 \\
\hline VoxelNet $^{[11]}$ & 81.98 & 65.46 & 62.85 \\
\hline SECOND $^{[12]}$ & 87.43 & 76.48 & 69.10 \\
\hline AVOD-FPN ${ }^{[20]}$ & 84.41 & 74.44 & 68.65 \\
\hline F-PointNet ${ }^{[16]}$ & 83.76 & 70.92 & 63.65 \\
\hline $\begin{array}{l}\text { PointRCNN }{ }^{[17]} \text { (无目标框 } \\
\text { 增强) }\end{array}$ & 88.45 & 77.67 & 76.30 \\
\hline 本文 & 89.08 & 78.04 & 77.05 \\
\hline
\end{tabular}
精度.

\section{4 可视化结果与分析}

为了验证候选区域生成网络可以学到结构信 息，本文在验证集上计算了结构信息真实值与预 测值的差距. 对于每一个点，计算它和真实值的误 差, 并统计平均误差. 如表 3 所示, $x, y, z$ 方向分 别表示车辆的宽度、长度和高度方向, 本文预测的 结构信息与真实值差距仅有 $8.50 \%$, 即每米的误差 为 $8.5 \mathrm{~cm}$, 证实了本文方法可以很好地预测结构 信息. 为了更直观地看到本文预测的结构特征, 将 预测的结构特征可视化为 RGB 颜色, 绿色框内为 真实值, 黄色框内为预测值, 并对黄色框进行平移 以方便对比. 图 4 中的可视化效果能够展示预测结 构信息的准确性.

\begin{tabular}{ccc} 
表 3 & 预测结构信息的平均误差 & $\%$ \\
\hline 方向 & 平均误差 \\
\hline$x$ & 7.69 \\
$y$ & 8.50 \\
$z$ & 9.31 \\
\hline 总体 & 8.50 \\
\hline
\end{tabular}

为了定性分析实验结果以及通过实验效果分 析本文方法, 图 5 给出本文方法得到的三维点云目 标检测的可视化效果. 其中, 绿色线框表示真实 


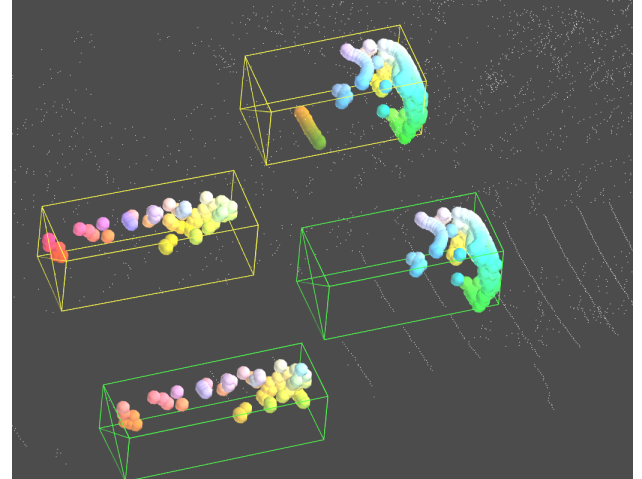

图 4 预测结构特征与真实结构特征可视化对比
框，黄色线框为本文检测结果，交叉线表示车辆的 朝向，红色为本文方法检测到但不存在于真实框 中; 图 5 下方为本文预测的结构信息. 本文中网络 输人没有图片，图片仅用来可视化. 其中大部分目 标都可以得到较高的 IoU. 一些失败情况可视化为 红色框, 如图 5 中间部分, 红色框中的车辆因为在 采集设备后方未被标记出来，但本文仍然能够根 据其结构信息将其检测出来. 左图中有一个真实 框没有被检测出来，是由于遮挡该车辆仅采集到 一个点，仅仅依靠点云几乎无法被检测到。

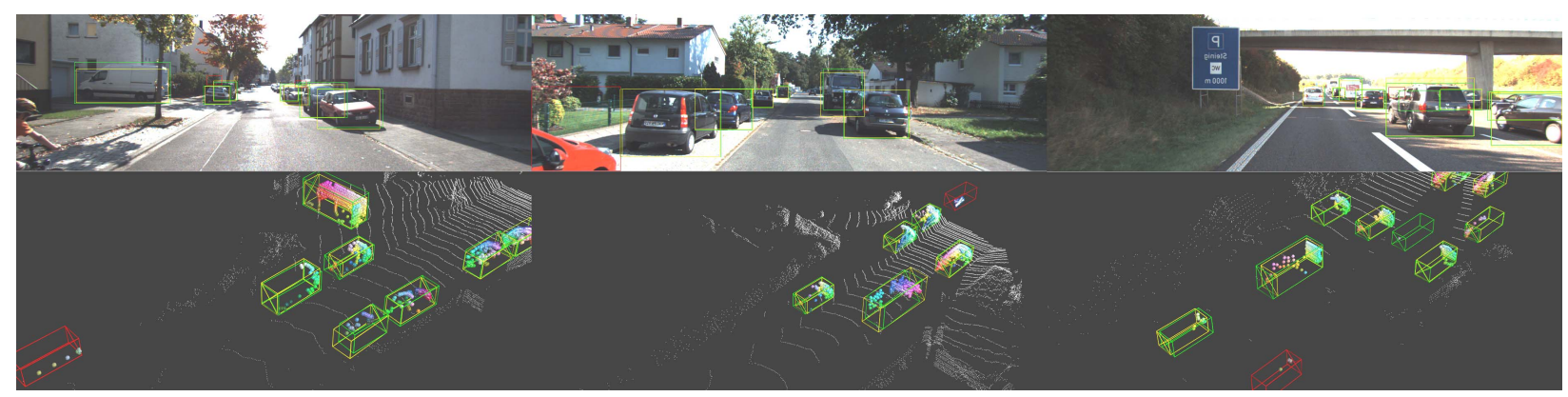

图 5 结构特征与检测结果可视化

\section{4 结 语}

本文针对 PointRCNN ${ }^{[17]}$ 中点云信息与目标检 测结果过渡太大的问题，提出了基于结构感知的 候选区域生成网络，充分利用了数据集的监督信 息, 使网络模型能够学习到介于点云和检测结果 的表示. 在 KITTI 数据集 ${ }^{[23]}$ 上进行三维点云候选 框生成和目标检测的实验结果表明，本文设计的 网络和训练流程极大地提高了候选框生成的质量, 同时提高了三维点云目标检测的精度，证明了其 有效性. 本文方法存在的不足为第 2 阶段精度提升 不显著. 下一步的工作重点是探究结构特征在目 标检测框架中嵌人的方式对结果的影响，从更深 的层次来理解其发挥作用的机制，进一步提升三 维点云目标检测的精度, 并尝试嵌人到其他模型, 验证其泛化能力.

\section{参考文献(References):}

[1] He K M, Zhang X Y, Ren S Q, et al. Deep residual learning for image recognition[C] //Proceedings of the IEEE Conference on Computer Vision and Pattern Recognition. Los Alamitos: IEEE Computer Society Press, 2016: 770-778

[2] Krizhevsky A, Sutskever I, Hinton G E. ImageNet classification with deep convolutional neural networks[J]. Communica- tions of the ACM, 2017, 60(6): 84-90

[3] Zhu H, Long M S, Wang J M, et al. Deep hashing network for efficient similarity retrieval[C] //Proceedings of the 30th AAAI Conference on Artificial Intelligence. Palo Alto: AAAI Press, 2016: 2415-2421

[4] Lin T Y, Goyal P, Girshick R, et al. Focal loss for dense object detection[C] //Proceedings of the IEEE International Conference on Computer Vision. Los Alamitos: IEEE Computer Society Press, 2017: 2999-3007

[5] Wang X L, Kong T, Shen C H, et al. SOLO: segmenting objects by locations[OL]. [2020-05-07]. https://arxiv.org/abs/ 1912. 04488v2

[6] Lang A H, Vora S, Caesar H, et al. PointPillars: fast encoders for object detection from point clouds[C] //Proceedings of the IEEE/CVF Conference on Computer Vision and Pattern Recognition. Los Alamitos: IEEE Computer Society Press, 2019: 12689-12697

[7] Yang B, Liang M, Urtasun R. HDNET: exploiting HD maps for 3D object detection[C] //Proceedings of the 2nd Conference on Robot Learning. Cambridge: MIT Press, 2018: 146-155

[8] Beltrán J, Guindel C, Moreno F M, et al. BirdNet: a 3D object detection framework from LiDAR information[C] //Proceedings of the 21st International Conference on Intelligent Transportation Systems. Los Alamitos: IEEE Computer Society Press, 2018: 3517-3523

[9] Yang B, Luo W J, Urtasun R. PIXOR: real-time 3D object detection from point clouds[C] //Proceedings of the IEEE/CVF Conference on Computer Vision and Pattern Recognition. Los Alamitos: IEEE Computer Society Press, 2018: 7652-7660

[10] Zhou J, Lu X Q, Tan X, et al. FVNet: 3D front-view proposal 
generation for real-time object detection from point clouds[C] //Proceedings of the 12th International Congress on Image and Sigal Processing, BioMedical Engineering and Informatics. Los Alamitos: IEEE Computer Society Press, 2019: 1-8

[11] Zhou Y, Tuzel O. VoxelNet: end-to-end learning for point cloud based 3D object detection[C] //Proceedings of the IEEE/CVF Conference on Computer Vision and Pattern Recognition. Los Alamitos: IEEE Computer Society Press, 2018: 4490-4499

[12] Yan Y, Mao Y X, Li B. SECOND: sparsely embedded convolutional detection[J]. Sensors, 2018, 18(10): 3337

[13] Shi S S, Wang Z, Shi J P, et al. From points to parts: 3D object detection from point cloud with part-aware and part- aggregation network[OL]. [2020-05-07]. https://arxiv.org/abs/ 1907.03670

[14] Charles R Q, Su H, Mo K C, et al. PointNet: deep learning on point sets for 3D classification and segmentation[C] //Proceedings of the IEEE International Conference on Computer Vision and Pattern Recognition. Los Alamitos: IEEE Computer Society Press, 2017: 77-85

[15] Qi C R, Yi L, Su H, et al. PointNet++: deep hierarchical feature learning on point sets in a metric space[OL]. [2020-05-07]. https://arxiv.org/pdf/1706.02413.pdf

[16] Qi C R, Liu W, Wu C X, et al. Frustum pointNets for 3D object detection from RGB-D data[C] //Proceedings of the IEEE/CVF Conference on Computer Vision and Pattern Recognition. Los Alamitos: IEEE Computer Society Press, 2018: 918-927

[17] Shi S S, Wang X G, Li H S. PointRCNN: 3D object proposal generation and detection from point cloud[C] //Proceedings of the IEEE/CVF Conference on Computer Vision and Pattern Recognition. Los Alamitos: IEEE Computer Society Press, 2019: 770-779

[18] Graham B, Der Maaten L V. Submanifold sparse convolutional networks[OL]. [2020-05-07]. https://arxiv.org/pdf/1706.01307. pdf

[19] Chen X Z, Ma H M, Wan J, et al. Multi-view 3D object detection network for autonomous driving[C] //Proceedings of the IEEE Conference on Computer Vision and Pattern Recognition. Los Alamitos: IEEE Computer Society Press, 2017: 6526-6534

[20] Ku J, Mozifian M, Lee J, et al. Joint 3D proposal generation and object detection from view aggregation[C] //Proceedings of the IEEE/RSJ International Conference on Intelligent Robots and Systems. Los Alamitos: IEEE Computer Society Press, 2018: $1-8$

[21] Ngiam J, Caine B, Han W, et al. StarNet: targeted computation for object detection in point clouds[OL]. [2020-05-07]. https:// arxiv.org/pdf/1908.11069.pdf

[22] Qi C R, Litany O, He K M, et al. Deep Hough voting for 3D object detection in point clouds[OL]. [2020-05-07]. https://arxiv.org/ pdf/1904.09664.pdf

[23] Geiger A, Lenz P, Urtasun R. Are we ready for autonomous driving? The KITTI vision benchmark suite[C] //Proceedings of the IEEE Conference on Computer Vision and Pattern Recognition. Los Alamitos: IEEE Computer Society Press, 2012: 3354-3361 